\title{
Data Mining Based Approach for Quality Prediction of Injection Molding Process
}

\author{
Dr.E.V.Ramana \\ Professor, Department of Mechanical Engineering \\ VNR Vignana Jyothi Institute of Engineering \&Technology, Hyderabad, India \\ email:egvramana@gmail.com
}

\begin{abstract}
Data mining techniques are gradually receiving attention in manufacturing area for process control, quality improvement and detecting causes of defects in products. Injection molding has been a challenge for manufactures and researchers to manufacture products at low cost due to the necessity of manipulating many process parameters in real time to meet the required specifications by keeping quality characteristics under control. Data mining approach has been adopted to predict the quality of product and identifying root causes of short shot and flash through data mining models created by applying Naïve Bayes, Decision Tree, Neural Net and Polynomial by Binomial Classification techniques. These models are trained on injection molding process dataset and their prediction accuracies are evaluated against test cases. Decision Tree, Polynominal by Binominal Classification and Neural Net models are found to have better prediction accuracy $(\mathbf{8 7 . 5 \% )}$ ) than Naïve Bayes Model $(75 \%)$ in predicting the quality of products. This approach can be used for fine tuning the process parameters by predicting the quality of product ahead of production to minimize the rejections.
\end{abstract}

Keywords: Data Mining, Naïve Bayes, Decision Tree, Neural Net, Injection Molding

\section{INTRODUCTION}

Injection molding has been one of the prominent methods of producing complex shaped plastic parts. The raw material is fed from hoper into the barrel where it is melted and then injected into the mold under pressure through nozzle. The plastic material is packed under pressure to compensate for shrinkage of molten plastic in the mold during its solidification. The part becomes rigid after cooling and ejected from the mold. The quality of part is influenced by various process variables such as barrel temperatures, nozzle temperature, injection pressure, time and speed, hold pressure and time, refill time, cooling time etc. These process variables are to be closely monitored and controlled to manufacture parts with desirable quality and minimizing the rejections.

Multistage Moving Window Generalized Regression Neural Network (GRNN), Recurrent Neural Network (RNN), Neuro-Fuzzy, Neural Network (NN) Support Vector Machine and Genetic Algorithm, Artificial Neural Networks (ANNs), Design of Experiments (DOE), Genetic Algorithms (GA) techniques are proposed for quality prediction, control and optimization of injection molding process [1-10]. Taguchi's parameter, regression analysis and Davidson-Fletcher-Powell method and evolutionary programming methods are used for optimal parameter setting of plastic injection molding [11-12]. In this paper, a data mining approach is implemented by applying Naïve Bayes, Decision Tree, Rule Induction, k Nearest Neighbor, Neural Net and Polynomial by Binomial Classification techniques in Rapidminer software for custom prediction of quality of a product (a cap for $50 \mathrm{ml}$ container) for a particular setting of process parameters and tracing the causes of short shot and flash.

\section{DATASET FOR RAPIDMINER SOFTWARE}

Injection molding process dataset of product (Cap for $50 \mathrm{ml}$ container) made of Polypropylene (PP) comprises of 38 records. Process attribute data - barrel temperatures at five zones; injection pressure, speed and time; hold pressure and time; refill time, cooling time, clamping force, percentage of masterbatch, cycle time attributes are collected. The dataset is divided into training and test datasets by randomly assigning 30 records to training set and remaining 8 records to test set. Test dataset is not involved in building the data models, but used for evaluating prediction accuracies of trained models.

\section{NAIVE BAYES CLASSIFIER}

Naive Bayes classifier is probabilistic classifier and assumes that all input attributes independently contribute to the probability of class label. The training (example) dataset is given as an input to Naive Bayes operator shown in the process presented in Fig.1. The model is delivered through output (mod) port of Naive Bayes operator subsequently given as an input to unlabelled (unl) port of Apply Model operator. This model can classify test dataset for prediction of the class label [13]. The labeled test dataset provided by Apply Model operator is given as input to Performance operator for performance evaluation of classification task. 


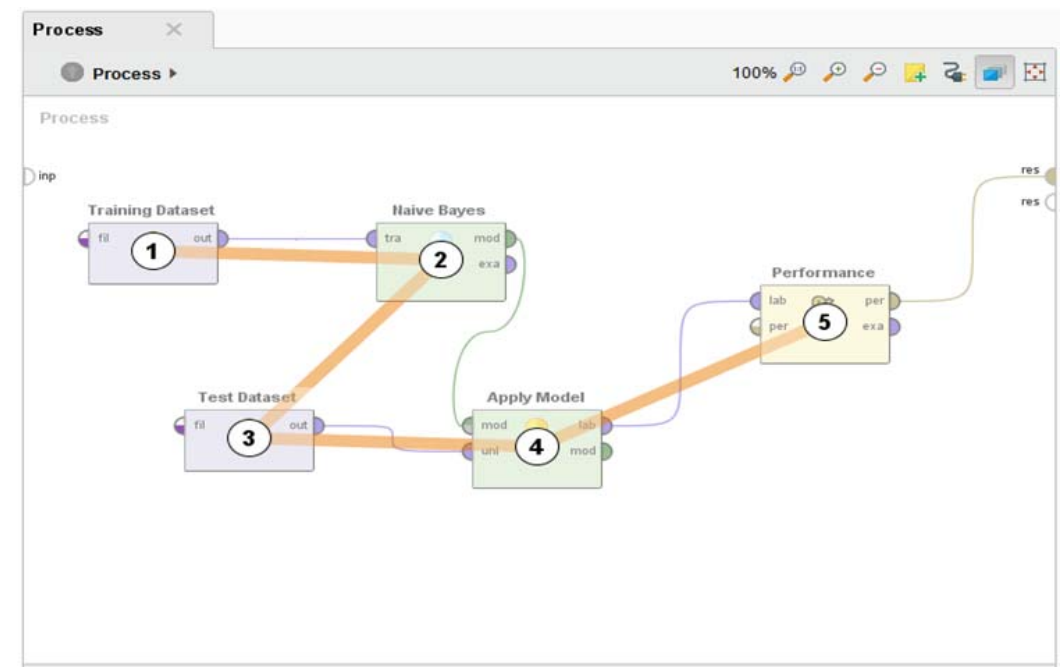

Fig.1. RapidMiner process with Naive Bayes Operator

The classification matrix generated by RapidMiner software along with class precision and recall and prediction accuracy of Naive Bayes model on test dataset is presented in Table I. The predicted class labels by Naive Bayes classifier along with probabilities are given in Table II.

TABLE I. Classification Matrix by Naive Bayes Model

\begin{tabular}{|l|c|c|c|c|}
\hline \multicolumn{5}{|c|}{ Accuracy 75.00\% } \\
\hline & True short shot & True Flash & True Acceptable & Class precision (\%) \\
\hline Pred. short shot & 2 & 0 & 0 & 100.00 \\
\hline Pred. Flash & 1 & 2 & 0 & 66.67 \\
\hline Pred. Acceptable & 1 & 0 & 2 & 66.67 \\
\hline Class recall & $50.00 \%$ & $100.00 \%$ & $100.00 \%$ & \\
\hline
\end{tabular}

TABLE II. True and Predicted Class Labels by Naive Bayes Classifier with Probabilities

\begin{tabular}{|c|l|l|c|c|c|}
\hline S.No & Classification & $\begin{array}{c}\text { Preditiction } \\
\text { (Classification) }\end{array}$ & $\begin{array}{c}\text { Confidence } \\
\text { (Acceptable) }\end{array}$ & $\begin{array}{c}\text { Confidence } \\
\text { (Short shot) }\end{array}$ & $\begin{array}{c}\text { Confidence } \\
\text { (Flash) }\end{array}$ \\
\hline 1 & Short Shot & Short Shot & 0 & 1 & 0 \\
\hline 2 & Short Shot & Acceptable & 1 & 0 & 0 \\
\hline 3 & Short Shot & Flash & 0 & 0 & 1 \\
\hline 4 & Short Shot & Short Shot & 0 & 1 & 0 \\
\hline 5 & Flash & Flash & 0 & 0 & 1 \\
\hline 6 & Acceptable & Acceptable & 1 & 0 & 0 \\
\hline 7 & Acceptable & Acceptable & 1 & 0 & 1 \\
\hline 8 & Flash & Flash & 0 & 0 & 0 \\
\hline
\end{tabular}

IV. DECISION TREE

A decision Tree is like an inverted tree with root at the top and grows downwards, where each internal node represents a test on an attribute, each branch denotes an outcome of test, and each leaf node holds a class label. It is easy to interpret the tree and rules can be derived from the tree. Pre-pruning is done during tree building process, where as post-pruning can be accomplished after building the tree.

Gain ratio is selected as criterion for splitting the attributes. Maximal depth parameter has been set as 5 to restrict the size of decision tree. Pre-pruning and post-pruning parameters are enabled and confidence level is set at 0.25 for pessimistic error calculation of pruning. The minimal gain is set at 0.1 and node is split if gain is greater than this value. Minimal leaf size parameter is set to 2 to ensure leaf node holds two examples in its subset. Minimal size for split is selected as 4 . Number of pre-pruning is set at 3, so that three alternative nodes are tried in case split is prevented by pre-pruning at any node [13-15].

The training dataset is given as an input to Decision Tree operator as shown in the process presented in Fig.2. The decision tree model and unlabelled test dataset are input to Apply Model operator through model and unlabelled ports. Decision Tree model builds the decision tree to classify test datasets for prediction of class 
label. The dataset with known class labels is given as input to Performance operator for performance evaluation of classification task.

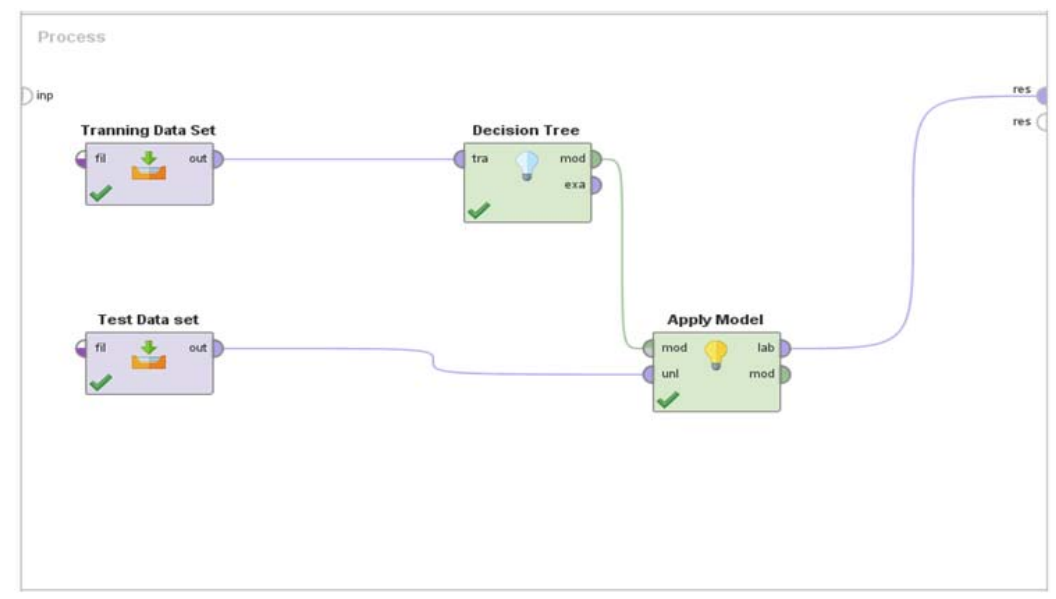

Fig.2. RapidMiner process with Decision Tree operator

The decision tree built by the model is presented in Fig.3.The classification matrix along with class precision and recall and prediction accuracy of Decision Tree model on test dataset is presented in Table III.

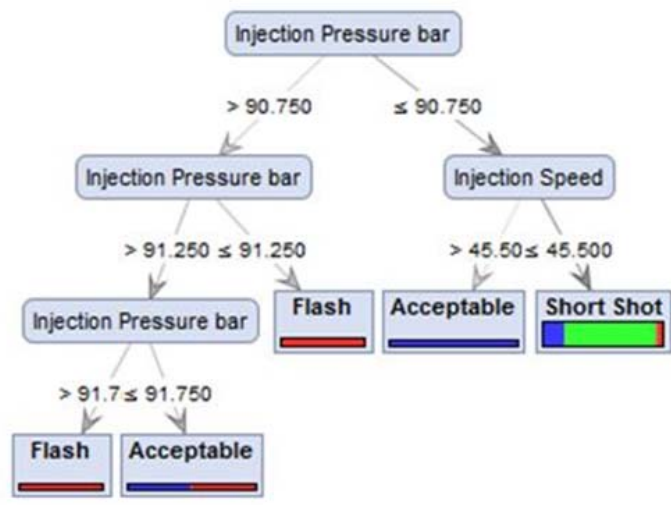

Fig.3 Decision tree

TABLE III. Classification Matrix by Decision Tree Model

\begin{tabular}{|l|c|c|c|c|}
\hline \multicolumn{5}{|c|}{ Accuracy 87.50\% } \\
\hline & True short shot & True Flash & True Acceptable & Class precision (\%) \\
\hline Pred. Short Shot & 4 & 1 & 0 & 80.00 \\
\hline Pred. Flash & 0 & 1 & 0 & 100.00 \\
\hline Pred. Acceptable & 0 & 0 & 2 & 100.00 \\
\hline Class recall & $100.00 \%$ & $50.00 \%$ & $100.00 \%$ & \\
\hline
\end{tabular}

\section{POLYNOMIAL BY BINOMIAL CLASSIFICATION MODEL}

Support Vector Machine (SVM) learner with Radial type kernel has been selected for classification task. SVM kernel gamma parameter is set at 1 after fine tuning to improve the performance of kernel. C-complexity constant value is set as 2 to generalize the model to avoid over-fitting or over-generalization. It cannot handle nominal attributes and can be applied on numeric attributes requiring nominal to numerical operator before applying this operator. The Polynomial by Binomial Classification operator is used to build polynomial classification model by using SVM as a binomial classification learner in its sub-process. It generates binomial classification models and aggregate responses of these models for classification polynomial label [13-15].

The polynomial classification model delivered from output port of Polynomial by Binomial Classification operator is given as input to Apply Model operator to predict the class label of test dataset. The labeled dataset delivered from output port of Apply Model operator is given as input to Performance operator for performance evaluation of the model to carry out the classification task [13]. The process involves Polynomial by Binomial Classification operator for classification task with SVM as its sub-process as presented in Fig.4. 


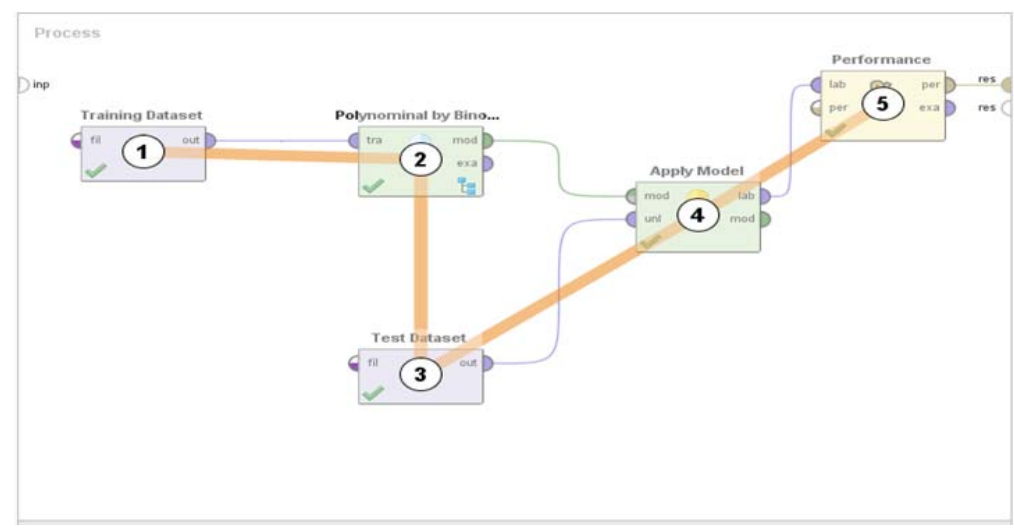

Fig.4 RapidMiner Process with Polynomial by Binomial Operator

The classification matrix along with class precision and recall and prediction accuracy of Polynomial by Binomial Classification model on test dataset is presented in Table IV.

TABLE IV. Classification Matrix - Polynomial by Binomial Classification Model

\begin{tabular}{|l|c|c|c|c|}
\hline \multicolumn{5}{|c|}{ Accuracy $87.50 \%$} \\
\hline & True short shot & True Flash & True Acceptable & Class precision (\%) \\
\hline Pred. Short Shot & 4 & 1 & 0 & 80.00 \\
\hline Pred. Flash & 0 & 1 & 0 & 100.00 \\
\hline Pred. Acceptable & 0 & 0 & 2 & 100.00 \\
\hline Class recall & $100.00 \%$ & $50.00 \%$ & $100.00 \%$ & \\
\hline
\end{tabular}

\section{NEURAL NET}

Neural network consists of a set of input and output units and each connection has a weight associated with it. The network learns by adjusting weights to correctly predict class label of input tuples. Neural networks involve long training times but highly tolerant of noisy data. Neural Network models can detect complex relationships existing between inputs and outputs and to find patterns in data.

Neural Net operator use a feed-forward neural network trained by back propagation algorithm. Polynominal attributes cannot be handled by this operator. Learning rate is set at a value of 0.3 which determines change of weights at each step. The value of momentum parameter is set as 0.2 . It adds a fraction of previous weight update to current one and help to prevent local maxima.

The training (example) dataset is given as an input to Neural Net operator shown in the process presented in Fig.5. The neural network model is delivered through output port of Neural Net operator subsequently given as an input to model (mod) port of Apply Model operator. The unlabelled test data set is given as input to second port (unl). Neural Network model can classify test data sets for prediction of the class label. The labeled test dataset provided by Apply Model operator is given as input to Performance operator for performance evaluation of classification task [13-14]. The classification matrix along with class precision and recall and prediction accuracy of Neural Net model on test dataset are presented in Table VI.

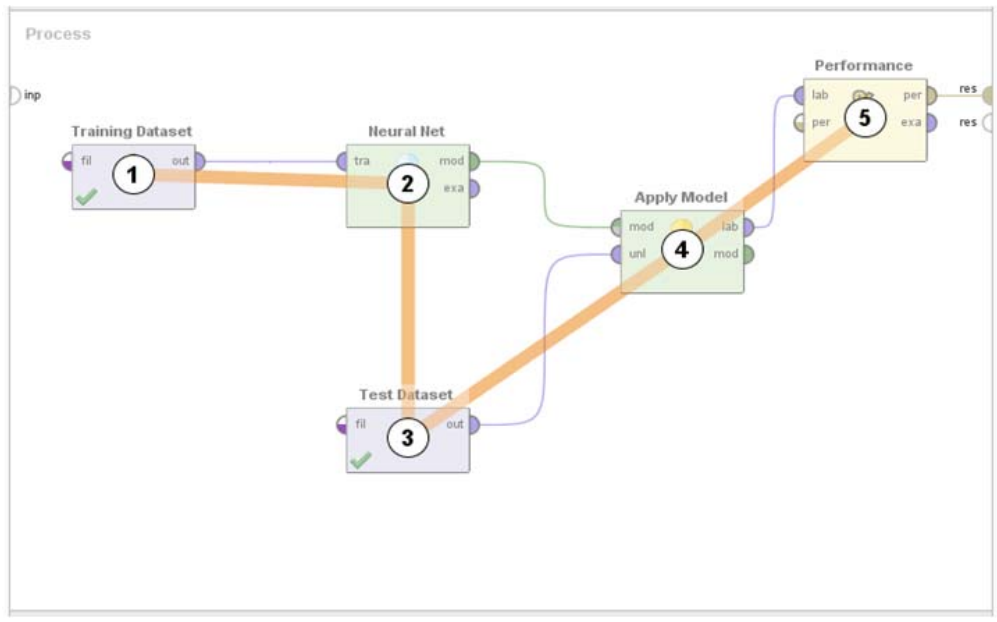

Fig.5 RapidMiner process with Neural Net operator 
TABLE VI. Classification Matrix - Neural Net Model

\begin{tabular}{|l|c|c|c|c|}
\hline \multicolumn{5}{|c|}{ Accuracy 87.50\% } \\
\hline & True short shot & True Flash & True Acceptable & Class precision (\%) \\
\hline Prediction short shot & 4 & 1 & 0 & 100.00 \\
\hline Prediction Flash & 0 & 1 & 0 & 100.00 \\
\hline Prediction Acceptable & 0 & 1 & 2 & 66.67 \\
\hline Class recall & $100.00 \%$ & $50.00 \%$ & $100.00 \%$ & \\
\hline
\end{tabular}

VII. CONCLUSION

The data mining models are built on the injection molding dataset by using Naïve Bayes, Decision Tree, Polynominal by Binominal classification and Neural Net techniques in RapidMiner software to make custom prediction of quality of products. These models are evaluated on test dataset for their prediction accuracies. Decision Tree, Polynominal by Binominal classification and Neural Net models are able to predict the class labels with an accuracy of $87.5 \%$ whereas the accuracy of Naïve Bayes model is $75 \%$. Decision Tree, Polynominal by Binominal classification and Neural Net models shall be used for custom prediction of quality of the chosen product in order to set process parameters to manufacture products of acceptable quality.

\section{REFERENCES}

[1] Guo, Xiao-ping, Fu-li Wang, and Shu Wang. Quality prediction and control of injection molding process using multistage MWGRNN method in Proceedings of the Fifth International Conference on Machine Learning and Cybernetics, Dalian: IEEE, 2006, pp3095-3100.

[2] Lu, Chi-Huang, Ching-Chih Tsai, Chi-Ming Liu, and Yuan-Hai Charng. Predictive control based on recurrent neural network and application to plastic injection molding processes in Industrial Electronics Society, 2007. IECON 2007. 33rd Annual Conference, IEEE, pp792-797.

[3] Hernandez, Pablo Ayala. Neural-Fuzzy Approach to Optimize Process Parameters for Injection Molding Machine in Intelligent Environments (IE), 2012 8th International Conference: IEEE, pp186-189.

[4] Yi, Mei, and Shan Zhi. The optimization of plastic injection molding process based on support vector machine and genetic algorithm in Intelligent Computation Technology and Automation (ICICTA), 2008 International Conference: IEEE, pp1258-1261.

[5] Hsieh KL, "Employing data mining technique to achieve the parameter optimization based on manufacturing intelligence", Journal of the Chinese Institute of Industrial Engineers, 24(4), pp309-318, 2007.

[6] Acharya UH, Mahesh C, "Winning Back the Customer's Confidence: A Case Study on the Application of Design of Experiments to an Injection-Molding Process”, Quality Engineering,11(3), pp357-363,1999.

[7] Deng YM, Zheng D, Sun BS, Zhong HD, “ Injection molding optimization for minimizing the defects of weld lines”, Polymer-Plastics Technology and Engineering,47(9), pp943-952, 2008.

[8] Wang L, Li Q, Shen C, Lu S, “Effects of process parameters and two-way interactions on sink mark depth of injection molded parts by using the design of experiment method", Polymer-Plastics Technology and Engineering, 47(1), pp30-35, 2007.

[9] Li Q, Li L, Si X, Rongji W, "Modeling the Effect of Injection Molding Process Parameters on Warpage Using Neural Network Theory", Journal of Macromolecular Science, (Part B), 54(9), pp1066-1080,2015

[10] Özek C, Çelık YH, “ Calculating molding parameters in plastic injection molds with ANN and developing software”, Materials and Manufacturing Processes, 27(2), pp160-168, 2012.

[11] Deng WJ, Chen CT, Sun CH, Chen WC, Chen CP, “ An effective approach for process parameter optimization in injection molding of plastic housing components", Polymer-Plastics Technology and Engineering, 47(9), pp910-919,2008.

[12] Jawaha S, Ramamoorthy P, "Dynamic optimization of injection molding process variables by evolutionary programming method" in Computer Communication and Informatics (ICCCI), 2012 International Conference, IEEE, 2012, pp1-6.

[13] RapidMiner 7 Operator Reference Manual, Rapidminer GmbH, 2016.

[14] Imam Tahyudin. Data Mining (Comparison Between Manual and Rapid Miner Process, Lap Lambert Academic Publishing, 2015.

[15] Jiawei Han, Micheline Kamber, Jian Pei. Data Mining Concepts and Techniques, Morgan Kaufmann Publishers, 2011.

\section{AUTHOR PROFILE}

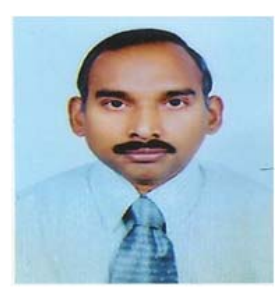

Dr. E. V. Ramana received his PhD degree in Mechanical Engineering in 2015 from JNTUH, Hyderabad. He received his M.Tech degree in Energy Systems and second M.Tech degree in CAD/CAM in the years 1992 and 1997 from JNT University, Hyderabad and P.G.D.C.A from Institute of Public Enterprise, Hyderabad. He possesses 20 years of experience in teaching and 6 years in industry and research and published many research papers in international journals, national and international conferences. $\mathrm{He}$ is currently working as a Professor of Mechanical Engineering, VNR Vignana Jyothi Institute of Engineering and Technology, Hyderabad and his active areas of research include Data Mining in Manufacturing, CAD/CAM, and automation. 